\section{AB1410-HPR COPING WITH INFLAMMATORY RHEUMATIC DISEASE AND FATIGUE: PATIENTS PERCEPTIONS OF THEIR NEEDS}

Margaret L. Søvik ${ }^{1,2}$, Tina Taule ${ }^{1}$, Ruth Else Mehl Eide1, Bjørg Rene ${ }^{1}$, Margaret Mary Strand ${ }^{3}$, Ingvill Devik ${ }^{4}$, Ingvild Kjeken ${ }^{5} .{ }^{1}$ Haukeland University Hospital, Department of Occupational Therapy, Bergen, Norway, ${ }^{2}$ Western Norway University of Applied Sciences, Department of Health and Functioning, Bergen, Norway, ${ }^{3}$ User representative, Bergen, Norway, ${ }^{4}$ Nordland Hospital, Bod, Department of Rheumatology, Bod, Norway; ${ }^{5}$ Diakonhjemmet Hospital, Department of Rheumatology, Norwegian National Advisory Unit on Rehabilitation in Rheumatology, Oslo, Norway

Background: Fatigue is difficult to manage. In addition to pain, fatigue is the symptom reported to have the most negative impact on performance of daily activities by people living with rheumatoid arthritis $(\mathrm{RA})^{1}$. At Haukeland University Hospital in Bergen, Norway, an activity pacing group is therefore offered as part of the rehabilitation program for people with inflammatory rheumatic diseases, to help them cope with fatigue in their everyday life.

Objectives: To explore patients experiences with the group and if there are unmet needs that should be addressed by health professionals.

Methods: Four to six months after attending rehabilitation, semi-structured interviews were conducted with two men and eight women of working age. Participants had a variety of inflammatory rheumatic diseases. The interviews were recorded and transcribed verbatim. Thematic analyses will be conducted.

Results: Preliminary findings indicate that persons living with rheumatic disease and fatigue have several needs to enhance their coping of everyday life, which can be described in three themes: (1) $A$ need for increased understanding of fatigue and recognition by health professionals: Several informants reported feeling concerned, insecure and worried because they were unable to understand why they experienced overwhelming tiredness. Consequently, they wanted more knowledge concerning to which degree their fatigue was caused by their inflammatory rheumatic disease, or if it was caused by something else. They also wanted the rheumatologists to pay more attention to fatigue in persons with rheumatic diseases. (2) A need for information sharing with peers: Such sharing enhanced their understanding of fatigue, motivated them to consider trying out new strategies to manage their fatigue, and seemed to be the most important outcome of the activity pacing group. (3) $A$ need for practicing specific self-management strategies: The informants valued that an occupational therapist led the group. They further described that the focus on activity balance and how to manage daily activities increased their awareness of what to do to manage their everyday life. Planning and prioritizing meaningful activities were the most frequently reported strategy. Despite gaining more knowledge, several of the informants seemed to struggle with changing their behavior and, thus, continued to exceed their energy reserves. Therefore, a few informants clearly expressed a need for follow-up over time and for practicing specific strategies, to be able to change their behavior.

Conclusion: The participants described seem to need information to increase their understanding of fatigue and how to manage their everyday life with fatigue. They also need rheumatologists to recognize fatigue experienced by persons with inflammatory rheumatic diseases. However, knowledge and increased awareness of self-management strategies alone do not seem to enable the participants to change their behavior. Therefore, there seems to be a need for follow-up allowing the participants to practice and evaluate goalsetting and the use of activity pacing strategies in their daily life.

\section{REFERENCE}

[1] Hewlett, S., et al., Patients' perceptions of fatigue in rheumatoid arthritis: overwhelming, uncontrollable, ignored. Arthritis Care \& Research, 2005. 53 (5): p. 697-702.

Acknowledgement: The authors would like to thank the patients who participated in this study.

Disclosure of Interests: None declared

DOI: 10.1136/annrheumdis-2019-eular.1019

\section{AB1411-HPR THE SELF- AND SHARED-MANAGEMENT OF PAEDIATRIC-ONSET RHEUMATIC AND MUSCULOSKELETAL DISEASES BY CHILDREN, YOUNG PEOPLE AND THEIR FAMILIES: INITIAL FINDINGS FROM A THEORY-GLEANING PROCESS WITH PATIENT ORGANISATION REPRESENTATIVES}

Simon Stones, ISMART Research Group. University of Leeds, School of Healthcare, Leeds, United Kingdom

Background: Encouraging children and young people (CYP) with rheumatic and musculoskeletal diseases (RMDs) and their families to adopt self-management behaviours from the point of diagnosis is likely to positively influence the long-term health and wellbeing of CYP. However, there is limited evidence of such interventions targeted at CYP and families.

Objectives: Therefore, the aim of this study was to identify What works, for whom, in what circumstances, and why.

Methods: The study was based on the logic of realist evaluation and the Individual and Family Self-Management Theory. Realist evaluation is a recognised model of theory-driven evaluation which enables an in-depth appreciation of how complex health interventions may or may not work in real-world settings. In this study, a realist evaluation was undertaken using qualitative research methods to explore and refine theories regarding self- and shared-management interventions. The realist evaluation began with a set of initial programme theories, which were initially tested through interviews with one group of stakeholders involved in providing self- and shared-management support. Interviews were transcribed intelligent verbatim and data were analysed in NVivo using a realist logic of analysis to identify contextual influencers, interventions mechanisms and outcomes.

Results: In order to refine and consolidate theories about the self- and shared-management of RMDs by CYP and their families, theory was gleaned from patient organisation representatives with experience of supporting CYP and families. Contextual influencers discussed included the characteristics of: i) individual CYP living with RMDs; ii) the family dynamic; iii) peer and social interaction among both CYP and families; iv) the educational environment; and v) Organisational processes and structures. Patient organisation representatives highlighted the family dynamic and navigating organisation processes and structures as key influencers on CYPs willingness and ability to begin their self-management journey in an age- and developmentally-appropriate manner.

Conclusion: Concepts gleaned during the first phase of the study, particularly around the influence of family dynamic, will be further tested, refined, and consolidated with other groups of stakeholders, including: CYP with RMDs; families; healthcare professionals from the paediatric rheumatology multi-disciplinary team; school staff; and patient organisation representatives. In time, it is anticipated that the findings will form a programme specification detailing what works, for whom, in what circumstances, and why with regards to the self- and shared-management of paediatric-onset RMDs.

\section{REFERENCES}

[1] Pawson, R. \& Tilley, N. (1997). Realistic Evaluation. London: Sage.

Acknowledgement: This work is supported by a University of Leeds School of Healthcare studentship.

Disclosure of Interests: Simon Stones Consultant for: SS has provided consultancy services to Envision Pharma Group, though this is not related to the contents of this abstract., Speakers bureau: SS has undertaken speaking engagements for Actelion, eyeforpharma, Four Health, Janssen and Roche, though these are not related to the contents of this abstract.

DOI: 10.1136/annrheumdis-2019-eular.6363 